\title{
MINI-UAV LIDAR FOR POWER LINE INSPECTION
}

\author{
G. E. Teng, M. Zhou, C. R. Li*, H. H. Wu, W. Li, F. R. Meng, C. C. Zhou, L. Ma
}

Key Laboratory of Quantitative Remote Sensing Information Technology, Academy of Opto-electronics, Chinese Academy of Sciences, 100094 Beijing, China - (tenggeer, zhoumei, crli, hhwu, liwei, mengfanrong, zhouchunch, malian)@aoe.ac.cn

KEY WORDS: Power Line Inspection, Mini-LiDAR, UAV, AOEagle, Pole, Transmission Line

\begin{abstract}
:
Light detection and ranging (LIDAR) system based on unmanned aerial vehicles (UAVs) recently are in rapid advancement, meanwhile portable and flexible mini-UAV-borne laser scanners have been a hot research field, especially for the complex terrain survey in the mountains and other areas. This study proposes a power line inspection system solution based on mini-UAV-borne LIDAR system-AOEagle, developed by Academy of Opto-Electronics, Chinese Academy of Sciences, which mounted on a Multi-rotor unmanned aerial vehicle for complex terrain survey according to real test. Furthermore, the point cloud data was explored to validate its applicability for power line inspection, in terms of corridor and line laser point clouds; deformation detection of power towers, etc. The feasibility and advantages of AOEagle have been demonstrated by the promising results based on the real-measured data in the field of power line inspection.
\end{abstract}

\section{INTRODUCTION}

As the Chinese economy flourishing the electricity demand is booming, therefore, the power transmission network and other electric infrastructure security work put forward higher requirements. For a long time, the inspection and maintenance of power line mainly rely on artificial field survey. This traditional power line inspection way costs a lot and can't ensure efficiency and safety. An efficient and flexible surveying scheme is needed to overcome this problem.

Light detection and ranging (LiDAR) recently has been developing quickly, for the reason of its accurate spatial point coordinates and flexibility on different platforms during depicting targets. Ground based LiDAR can not only guarantee a large number of points coverage and have a major detecting radius, but the ground LiDAR system is too heavy, for special applications such as mountain power line inspection and the precipitous terrain forest resources survey, whose human costs and cumbersome character are the bottlenecks. Meanwhile, Mobile laser scanning (MLS) as a settlement offering high density of data also suffers from the issue of no entry to the steep mountains and dense forest (Lin, 2011).Airborne laser scanning (ALS) has been extensively developed and also employed in a variety of special applications in recently years, but considering its high costs and vulnerability in montane area, ALS is not an optimal method.

Unmanned aerial vehicles (UAVs) as alternative platforms for laser scanning provide a good choice to overcome the aforementioned issue. Currently, many researches have been done, from preliminary schematics and prototypes of UAV-borne LiDAR systems to more comprehensive systems (Lin, 2011). However, the aforementioned equipment is not appropriate to be carried into steep montane areas and deep forests manually for the reasons of their weight.
Mini-UAVs seem to be a natural choice as the supplementary solution. Installed with INS and GPS, mini-UAV-borne LIDAR systems can further act as a promising mapping plan, which can deploy efficient, accurate, and flexible surveying projects (Lin, 2011). Zhao installed a remotely controlled helicopter with GPS navigation sensors and a laser altimeter for topographic surveys (Zhao, 2006). Geske had developed a miniature laser range altimeter for autonomous navigation and landing in small UAVS (Geske, 2008). Lambers tried to combine the mini-UAV optical images with laser scanning performed on the conventional platforms (Lambers, 2007). Hyyppä made a review of the application of airborne laser scanning in the northern woodland (Hyyppä, 2008).

This paper presents an advanced mini-UAV-borne LiDAR system-AOEagle, which is developed in the Academy of Opto-Electronics, Chinese Academy of Sciences. Based on the real-measured point clouds, the applicability and flexible for power line inspection of AOEagle are demonstrated in terms of corridor and line laser point clouds extraction; tower and line extraction; deformation detection of power towers; power line span and sag detection and dangerous points detection.

\section{EQUIPMENT INTRODUCTION}

\subsection{AOEagle System}

In the configuration of mini-UAV-borne AOEagle system, the instruments are mounted under a radio-controlled six-rotor unmanned aerial vehicle, as shown in Figure 1. The whole system weights $9 \mathrm{~kg}$ (UAV and AOEagle), maximum time of endurance is 40 minutes, and the size of vehicle after folded is: $543 \mathrm{~mm} * 460 \mathrm{~mm} * 558 \mathrm{~mm}$. The AOEagle frame is isolated from the vehicle to eliminate high-frequency vibrations. AOEagle is mainly made up of a laser scanner and a GPS/INS 
unit. Main technical parameters of AOEagle are listed in Table 1 .

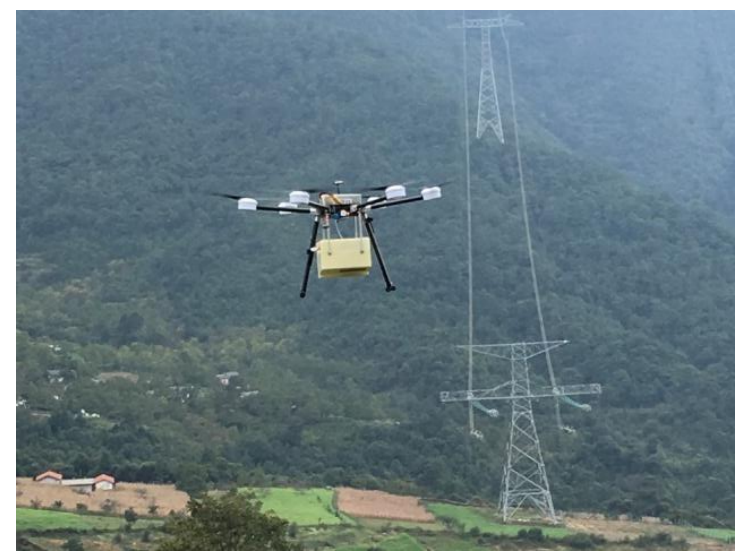

Figure 1. Power line inspection

\begin{tabular}{|l|c|}
\hline Item & Description \\
\hline Weight & $3.5 \mathrm{~kg}$ \\
Power Consumption & $13 \mathrm{~W}$ \\
Angular Resolution & $0.125^{\circ}$ \\
Maximum Range & $200 \mathrm{~m}$ \\
Distance & $110^{\circ}$ \\
Detection Angle & $4 \mathrm{~cm}$ \\
Range Resolution & $905 \mathrm{~nm}$ \\
Wavelength & $0.08^{\circ}$ \\
Azimuth Accuracy & $0.015^{\circ}$ \\
Pitch Accuracy & $0.015^{\circ}$ \\
Roll Accuracy & $2 \mathrm{~cm}$ \\
Horizontal Accuracy & $2 \mathrm{~cm}$ \\
Vertical Accuracy & \\
\hline
\end{tabular}

Table 1. Specifications of the AOEagle

\subsection{Experiment}

A test flight was deployed in Xichang, Sichuan, China, on March 14, 2017, and the point cloud obtained in this experiment after processing; generate the point cloud in the Cartesian coordinate system. Vehicle run under manual control, specific working procedures are as following: fast rectilinear flight and circuit flight like "8" are necessary procedures before UAV inspects the power line, which improves the GNSS alignment accuracy as forward calculating references; real-time images are transferred to the pilots by a camera installed on the steering gear through the LightBridge2, according to the real-time images function, UAV flies above the power tower height (entire tower can be seen in the screen); pilots controls the UAV to fly over the tower and adjusts the height; inspecting the power line along the power line. After inspection, circuit flight like " 8 " and fast rectilinear flight are still need to be done as reverse calculating references.

AOEagle could offer differential GPS and precise point position two-level products on the basis of different demands. The altitude of this experiment from $2800 \mathrm{~m}$ to $3000 \mathrm{~m}$ (above ground varied between 50 and $150 \mathrm{~m}$ ), point density ranged from 70 to 200 points per square meter depends on the environments. Based upon the China's power industry standard "Operating code for overhead transmission line DLT741-2010", as shown in Table 2, boundary distances need to be investigated based on the voltage levels of transmission lines. Considering that the targets are $800 \mathrm{kv}$, we expend the width (boundary distance to the power line) to $30 \mathrm{~m}$ on both sides of the corridor.
One other thing to be noted is that the distance between the camera and the antenna of GPS have to be more than $30 \mathrm{~cm}$, otherwise, the latter will be unable to search signal from satellites.

\begin{tabular}{|c|c|}
\hline Voltage Level & Edge Distance \\
\hline $\mathrm{kV}$ & $\mathrm{m}$ \\
$66-110$ & 10 \\
$220-330$ & 15 \\
500 & 20 \\
750 & 25 \\
\hline
\end{tabular}

Table 2. Edge distance of voltages in general area

\section{METHODS AND APPLICATIONS}

According to the long-term manual inspection experience of local power maintenance staff and "Operating code for overhead transmission line DLT741-2010", this paper performed the following works based on the data obtained by AOEagle: corridor and line point cloud extraction, pole and transmission line point cloud extraction, pole deformation detection, span and sag detection, dangerous point detection. Point cloud post-processing flow has been showed in Figure 2 based on the commercial software Inertial Explorer 8.60. Meanwhile, we demonstrated the feasibility and advantages of AOEagle in the field of power line inspection based on the TerraScan.

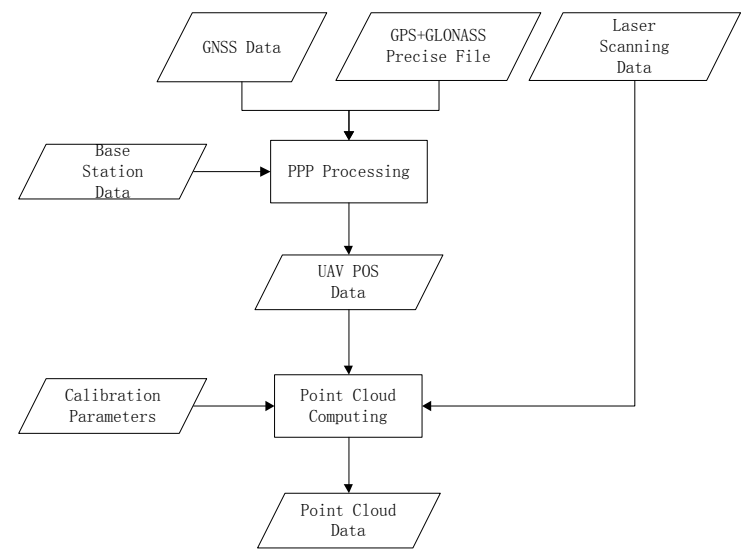

Figure 2. Point cloud post-processing flow

\subsection{Corridor and Line Point Cloud Extraction}

As the experiment area is JinSu transmission line, whose voltage is $800 \mathrm{kV}$. We find a new inspection paradigm that balances safety and $30 \mathrm{~m}$ corridor width with the characteristics of the AOEagle. The flight height is $18 \mathrm{~m}$ above the lightning line (the top line). Some of the ground points were loss, no doubt, because the maximum ranging distance is $200 \mathrm{~m}$, just as flight path shown in Figure 3. Figure 4 and Figure 5 are the top view and side view after classification, respectively. According to the corridor and line point cloud extraction views, we could observe the operation surroundings and inspect the hidden dangers preliminarily. 


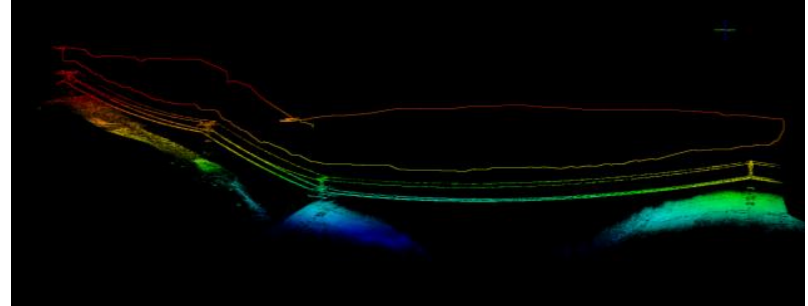

Figure 3. Jinsu transmission line No.80-83 tower flight path

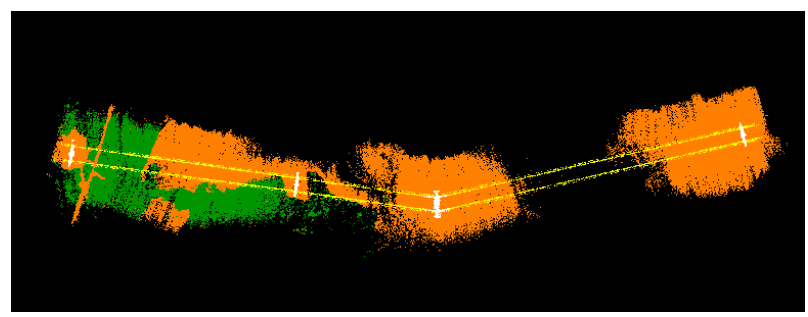

Figure 4. Top view of corridor and line laser point cloud

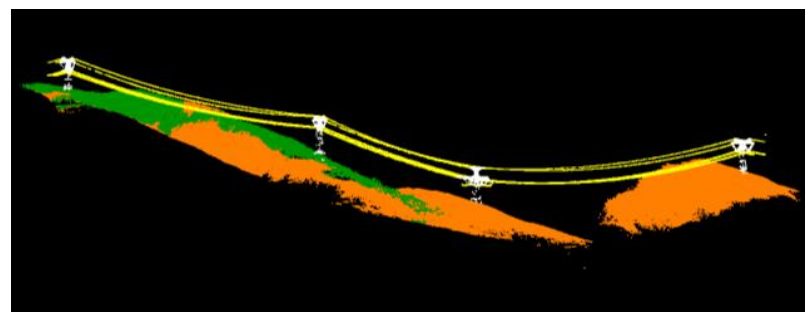

Figure 5. Side view of corridor and line laser point cloud

\subsection{Pole and Transmission Line Point Cloud Extraction}

In this paper, we extracted the tower and transmission line point cloud to eliminate the impact of the ground points, just as shown in Figure 6. Features extraction facilitates the inspection of the tower structure and transmission lines destruction, break and other potential security risks.

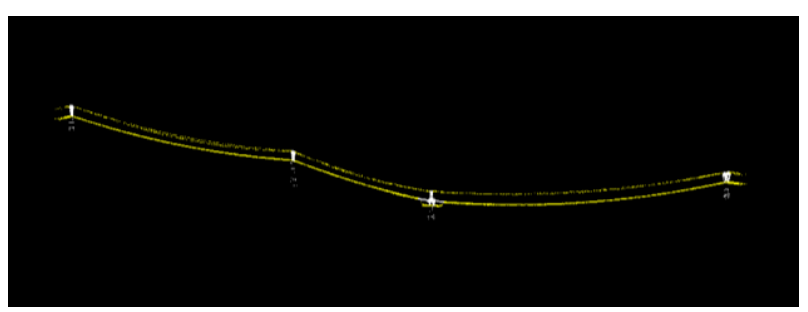

Figure 6. Features extraction

\subsection{Pole Deformation Detection}

Based on the accurate three-dimensional coordinate of the LiDAR, the pole leaning, pole main structure bending, timbering deformation and so on can be detected, just as shown in Figure. 7. This paper compared the AOEagle measured pole height $(53.3317 \mathrm{~m})$ with the total station measured height $(53.35 \mathrm{~m})$ in the field, and the error is within $\pm 0.02 \mathrm{~m}$, in Figure 8 . The results can satisfy the angle-steel pole leaning resolution requirements $(0.5 \%)$ in "overhead Transmission line operating specifications DLT741-2010" section 5.1.2. In this way, we could check out the pole leaning, settlement and other deformation easily and intuitively.

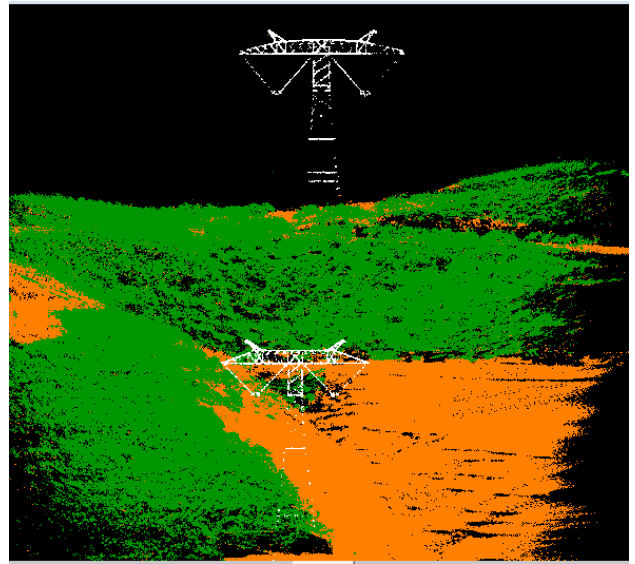

Figure 7. Pole extraction

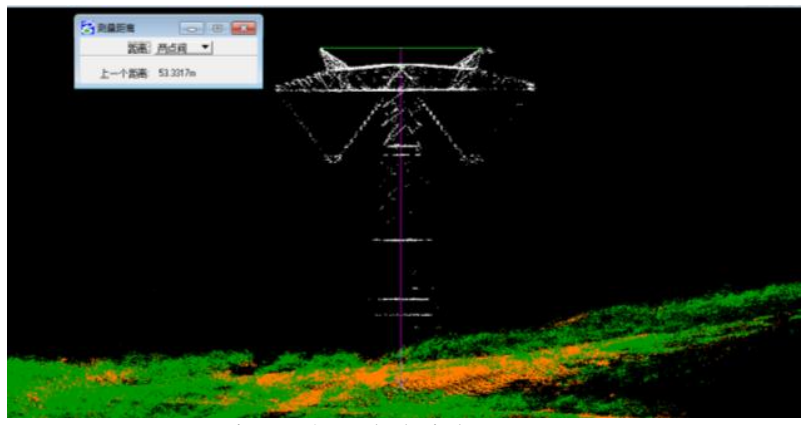

Figure 8. Pole height measurement

\subsection{Span and Sag Detection}

The measurement of the span of the tower could facilitate the calculation process of the distance between the nearby poles and measure the inclination angle of poles in steep areas. In this paper we calculated the distance between No. 80 and No. 81 (poles base center), in Fig. 9. The error between AOEagle data $(388.7027 \mathrm{~m})$ and field observed data by total station data (388.66m) is within $\pm 0.05 \mathrm{~m}$, and the relative error is $1 / 7773$. This confirms the practicality of the AOEagle in the span detection.

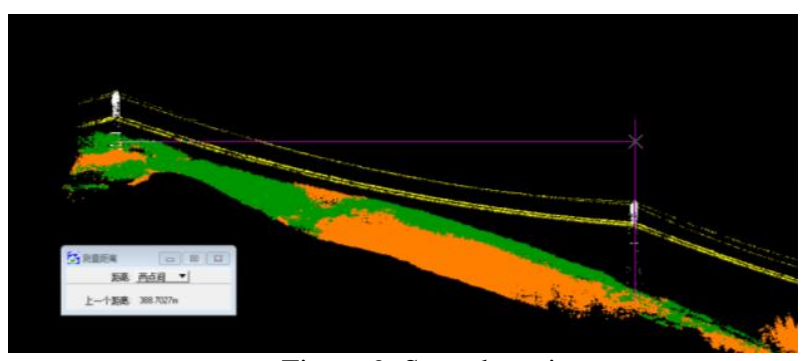

Figure 9. Span detection

According to the measurement of the distance between the lowest conductor point and the assumed straight line connecting two poles, detecting and recording the transmission line conditions in different temperatures and humidity becomes convenient. Furthermore, cable pulling analysis can also be done by sag detection. 


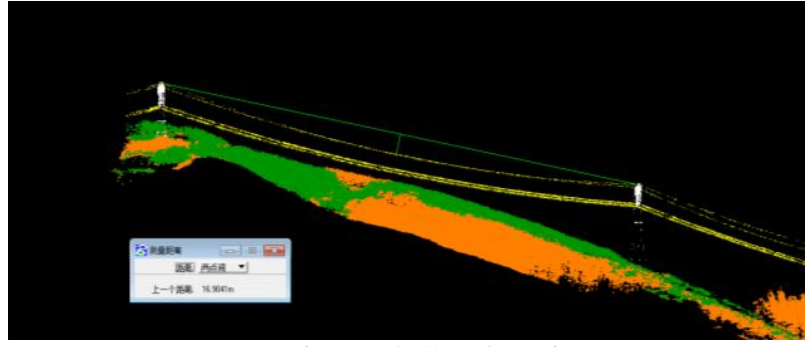

Figure 10. Sag detection

\subsection{Dangerous Point Detection}

Ground features, hills, buildings and trees are potential safety hazards of transmission line, detecting these dangerous points is the focus of power line inspection. According to the safe distance requirements in the "overhead transmission line operating specifications DLT741-2010" appendix A1-A10, this paper excluded a potential dangerous point in JinSu transmission line No.80-83 poles, just as shown in Figure 11, by measuring the minimum distance between the Transmission Line Class and the Ground Class/Plant Class with the usage of the TerraScan.

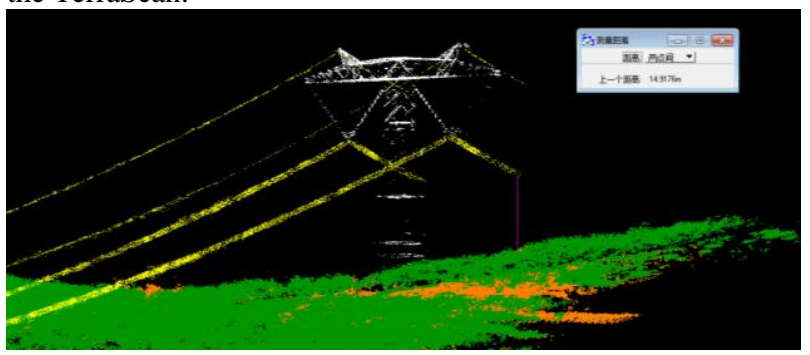

Figure 11. Dangerous point detection

\section{DISCUSSION AND CONCLUSIONS}

Based on the applications of the aforementioned power line inspection test, AOEagle's performance has been well verified, especially in the mountains. AOEagle is advantageous in terms of price and agility. High-precision ground resolution and high operating efficiency also validate the wide application prospects of Mini-LiDAR in power line inspection field.

AOEagle is not limited to unmanned aerial vehicles (UAVs), which can also be mounted on the car and other platforms. Furthermore, AOEagle can be integrated with other devices, such as CCD camera. AOEagle's performance in other areas, such as 3D city modeling and forestry resources, will be discussed in the next steps.

\section{ACKNOWLEDGEMENTS}

The work is supported by the National International Science and Technology Cooperation Project (Grant No.2015DFA70930).

\section{REFERENCES}

Hyyppä, J., 2008. Review of methods of small footprint airborne laser scanning for extracting forest inventory data in boreal forests. International Journal of Remote Sensing, 29:5, pp. 1339- 1366.

J. Geske, 2008. Miniature laser range finders and laser altimeters. In Proc. IEEE Conf. Avionics Fiber-Opt. Photo. Technol, pp.53-54.
K. Lambers, 2007. Combining photogrammetry and laser scanning for the recording and modelling of the Late Intermediate Period site of Pinchango Alto, Palpa, Peru. $J$. Archaeol. Sci., vol. 34, no.10, pp. 1702-1712.

M. Nagai, 2009. UAV-borne 3-D mapping system by multisensory integration. IEEE Trans. Geosci. Remote Sens., Vol. 47, no.3, pp. 701-708.

P. Johnson, 2006. Unmanned aerial vehicle as the platform for lightweight laser sensing to produce sub-meter accuracy terrain maps for less than 5/km2, Mech. Eng. Dept., Columbia Univ., New York.

X. Zhao, 2006. A remote aerial robot for topographic survey," in Proc. IEEE Int. Conf. Intel. Robots Syst, pp. 3143-3148.

Y. Lin, 2011a. K-segments based geometric modelling of VLS scan lines, IEEE Geosci. Remote Sens. Lett., vol.8, no. 1, pp. 93-97

Y. Lin, 2010b. From TLS to VLS: Biomass estimation at individual tree level, Remote Sens., vol.2, no. 8, pp. 1864-1879

Y. Lin, 2011c. MINI-UAV borne LiDAR for fine scale mapping. IEEE Geosci. Remote Sens. Lett., vol.8, no. 3. pp. 426-430 